\title{
Arthroscopic Management of Meniscal Root Injury
}

\author{
Younus Akl, Emad Zayed, Mahmoud Maher
}

Department of Orthopedic Surgery, Faculty of Medicine, Al-Azhar University, Cairo, Egypt

Corresponding author: Mahmoud Maher,email: mahercordoba@gmail.com

\begin{abstract}
Background: Meniscal root tear s are becoming increasingly recognized. They can cause rapidly progressive arthritis. Purpose: The purpose of this study was to document the radiological and functional outcome of arthroscopic management of meniscal root tears injuries. Patients and Methods: This study was held on 20 patients suffered from meniscal root tears at Al-Azhar University Hospitals. There were 11 males and 9 females with mean age 41.63 (2350) years old. All patients were evaluated preoperatively and postoperatively with mean follow up 6 months.

Results: Clinical outcome measures significantly improved after surgery. Preoperatively there were 13 patients $(65 \%)$ had poor score and 7 patients (35\%) had fair score. Postoperatively there were 3 patients (15\%) had poor score, 4 patients (20\%) had fair score, 6 patients (30\%) had good score and 7 patients $(35 \%)$ had excellent score. The mean IKDC score for meniscal repair increased, and the mean IKDC score for partial meniscectomy increased. Postoperative MRI showed complete healing in 12 patients and failure in 8 patients.preoperative ME was $>3 \mathrm{~mm}$ in 17 patients and $\leq 3 \mathrm{~mm}$ in 3 patients .postoperative ME was $\leq 3 \mathrm{~mm}$ in 11 patients and $>3 \mathrm{~mm}$ in 9 patients. Conclusions: Meniscal root repair has increased in popularity and when done in selected patients results in a high rate of healing and restoring the ability of the meniscus to dissipate axial tibiofemoral loads, thereby slowing or halting arthritic progression.
\end{abstract}

Keywords: Root tear injury, partial menisectomy, pullout suture repair.

\section{INTRODUCTION}

The meniscus roots are responsible for meniscal stability, which is necessary for proper meniscal function. Without the meniscus root attachments, the meniscus would be unstable ${ }^{(1)}$.

A meniscus root tear is described as a radial tear or avulsion at the posterior horn attachment to bone for medial or lateral meniscus ${ }^{(2)}$.

Medial meniscal tears are common in patients with chronic ACL insufficiency, while lateral meniscal tears is found in acute ACL injuries (3). Specifically, meniscal root tears result in a loss of hoop stress, as well as functional load distribution, exposing the articular cartilage to abnormal forces that are comparable to those following total meniscectomy ${ }^{(4)}$.

MRI diagnosis of root tear is based on the presence of radial tears on the axial plane, signs of truncation and extrusion on the coronal plane, and the ghost sign from the sagittal plane ${ }^{(5)}$.

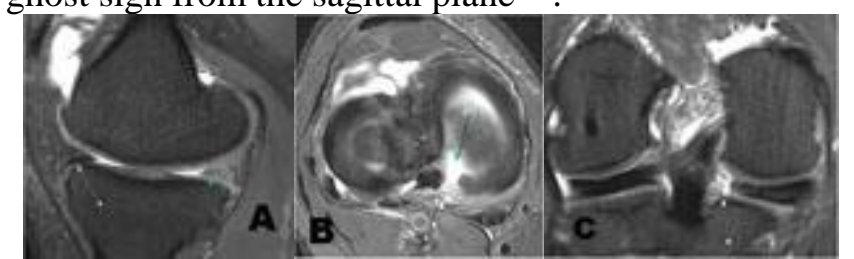

Figure (1): Images - MRI with knee coil. A - Ghost sign- sagittal plane. B - Radial linear defect in axial plane. C- Vertical linear defect in coronal plane.

Treatment of root tear is partial meniscectomy for degenerative tear ${ }^{(6)}$. In comparison, root tear repair focuses on restoring hoop tension and preventing progression to degenerative arthritic changes. The repair methods can be divided into pull-out suture ${ }^{(7)}$ or suture anchor techniques ${ }^{(8)}$.

Arthroscopic partial meniscectomy is normally effective in eliminating mechanical and irritative symptoms associated with a damaged meniscus ${ }^{(9)}$.

\section{PATIENTS AND METHODS}

This prospective cohort study was approved by the Ethical Committee of Al-Azhar University. A total of 20 patients with root tears were treated in Al-Azhar University Hospitals using arthroscope. All patient were signed an informed consent form.

We include patients with meniscal root tears either traumatic or degenerative.

Exclusion criteria were: a) elder patients, b) poor surgical candidates, c) initial bone marrow edema because of change loading in the compartment greater varus mechanical axis deviation.

There were 11 males and 9 females with mean age $41.63 \pm 9.7$ and range 23 to 50 years old.

All patients had preoperative clinical assessment in the form of general and local examination and special tests as Payr's test and McMurray test.
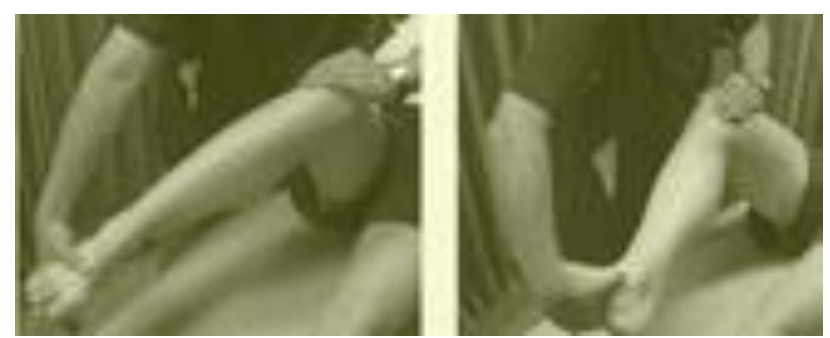

Fig (2): Mcmurrays test. The tibia rotated on the femur in to full internal rotation and (fig b) rotated in to full external rotation ${ }^{(10)}$.

MRI was done as routine preoperative investigation to assess meniscal root tear.

All patients were assessed preoperative by Lysholm and IKCD score.

Operative technique:

Surgical technique:

All the patients were anaesthetized by spinal anesthesia. Patients were placed in the supine position. 
A routine arthroscopic examination of the knee joint was performed through the standard anterolateral and anteromedial portals with a distended knee joint using the arthroscopic infusion pump.

\section{Partial menisectomy:}

It's often easier to resect the worn parts and fragments with the shaver fiest in order to improve visualization then, the main fragments can be better identified and treated.

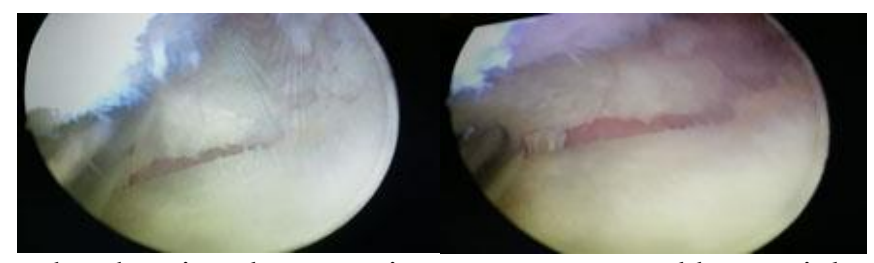

Fig (3): Arthroscopy photography showing degenerative root tear treated by partial menisectomy.

\section{Pull out suture repair:}

Surgical technique:

Confirmation of the lesion by arthroscopic examination showing posterior root tear of medial meniscus.

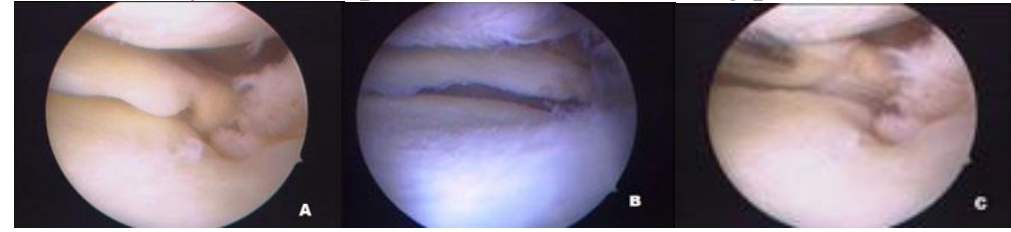

Fig. (4): A, B and C Arthroscopic photography showing posterior root tear of medial meniscus.

After confirmation of a lesion, the footprint was confirmed by removing the cartilage of the tibial plateau, which was attached to the posterior root of the medial meniscus, with a curette and round bur.

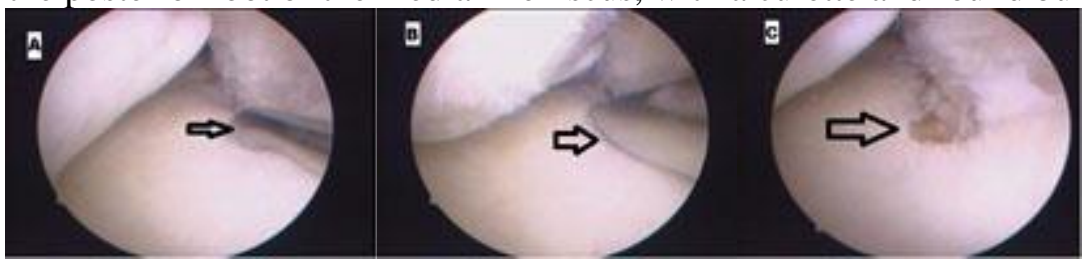

Fig. (5): A, B and C Arthroscopic photography from antromedial portal showing decortication of the footprint.

Under visualization through the anteromedial portal, the anterior cruciate ligament tibial aiming guide with a short tip was introduced through the anterolateral portal. The tip of the guide with $40^{\circ}$ to $45^{\circ}$ was placed at the decorticated footprint of the posterior root of the medial meniscus. The sleeve of the guide was fixed on the anterolateral cortex of the proximal tibia distal to the gerdy`s tubercle after making of a $2 \mathrm{~cm}$ vertical incision.

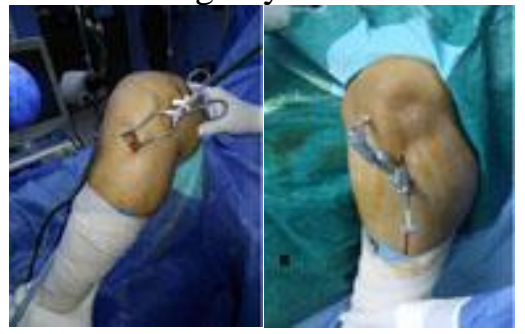

Fig. (6): Intraoperative picture: A, $2 \mathrm{~cm}$ vertical incision was done on the antrolateral cortex of tibia and insertion of ACL aiming guide.

The entry point of tibial anterolateral cortex was just below jerdy`s tubercle on the lateral aspect of the tibia. A guide pin was drilled from the the anterolateral cortex of proximal tibia to the insertion site of the posterior root tear site of the medial meniscus through the sleeve.

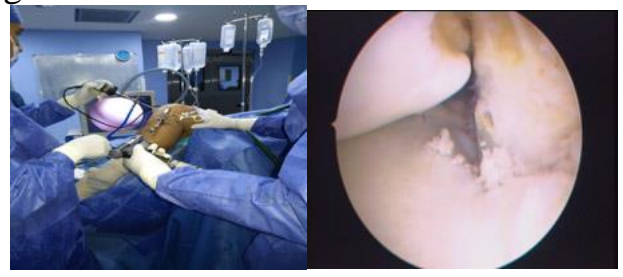

Fig. (7): Intraoperative photography showing a guide pin is introduced through the sleeve of the anterior cruciate ligament tibial aiming device to the footprint.

A tibial tunnel was made from the anterolateral cortex of the proximal tibia to the footprint of the posterior root of the medial meniscus by use of a guide pin with a $4.5 \mathrm{~mm}$ drill bit. 


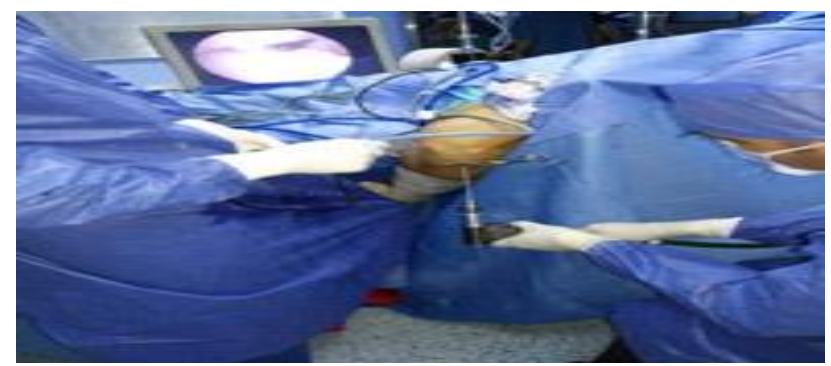

Fig. (8): Intraoperative photography showing making of a tibial tunnel.

A knee scorpion suture passer loaded with No.2-0 suture material was inserted through the anteromedial portal. The detached root portion of the medial meniscus posterior horn was penetrated from the tibial surface to the femoral surface of the meniscus in a vertical direction.Then the No.2 fibrwire suture was shuttelled on no. 2-0 suture through the meniscus posterior root.
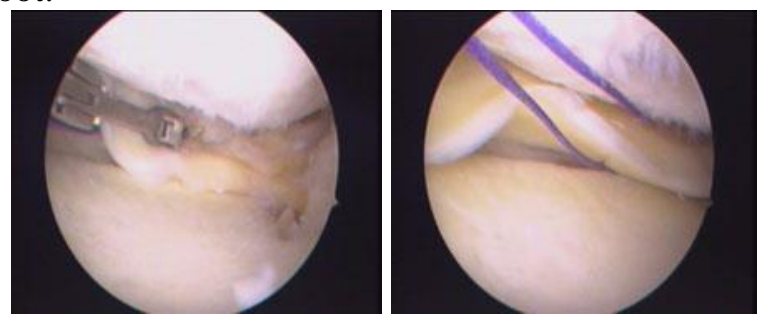

Fig. (9): Arthroscopic photography from antrolateral portal showing a knee scorpion suture passer loaded with No.20 suture material was inserted through the anteromedial portal.

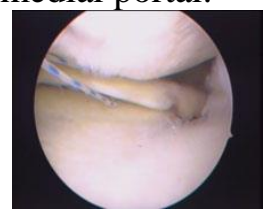

Fig. (10): Arthroscopic photography from antrolateral portal showing No. 2 fibrewire was shuttelled on no. 2-0 suture

No. 2-0 suture material was passed in the same manner in a more peripheral site to the prior suture, a ring grasper was used to retrive the two upper limbs of both fibrewire suture and no 2-0 suture material. Then the fibrewire was shuttelled into the meniscus root using the no. 2-0 suture material.

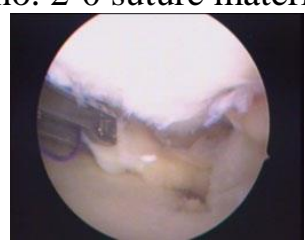

Fig. (11): Arthroscopic photography from antrolateral portal showing No. 2-0 suture material was passed in the same manner in a more peripheral site to the prior suture.

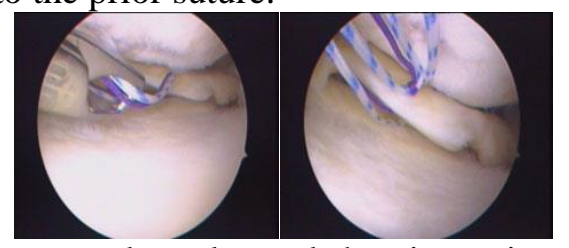

Fig. (12): Arthroscopic photography from antrolateral portal showing a ring grasper was used to retrive the two upper limbs of both fibrewire suture.

This suture is working as a tension suture; a ring grasper was advanced through the tibial tunnel. The tension suture was retrived through the tibial tunnel to the tibial surface.

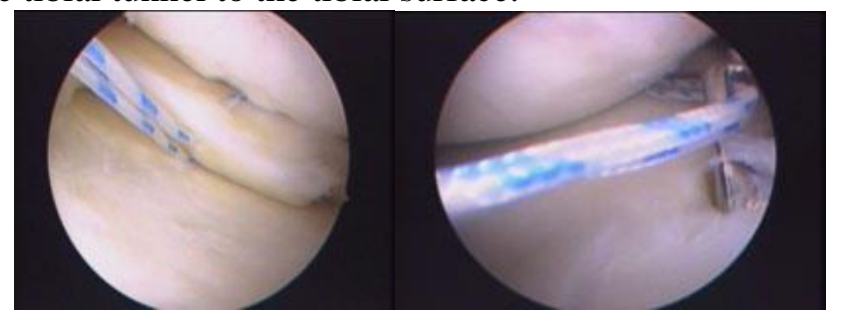


Fig. (13): Arthroscopic photography from antrolateral portal showing a tension suture.

In some cases a spinal cannula was used to advance a suture material into the meniscus root through the tibial tunnel, in another cases lasso suture passer was used from the anteromedial portal to pass a suture material to perform a shuttle but we found knee scorpion is more easier and less time consuming.

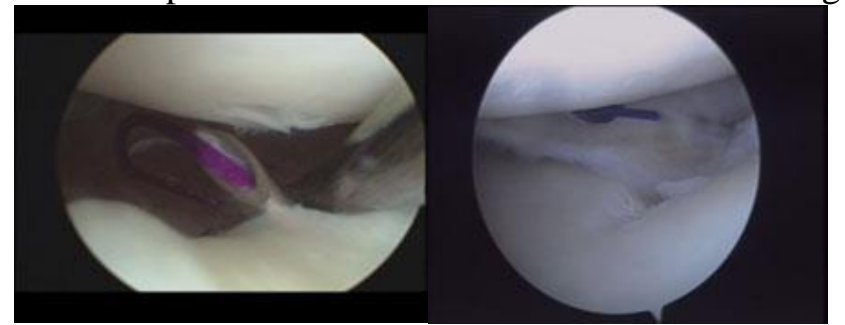

Fig. (14): Arthroscopic photogarphy showing a spinal cannula other case using lasso suture passer.

Another 2-0 suture material was loaded, passed half centimeter medial to the tension suture. Then, no 2 fibrewire was shuttelled into the meniscus. Again, no 2-0 was passed in the peripheral zone of the meniscus in line with the second fibrewire.

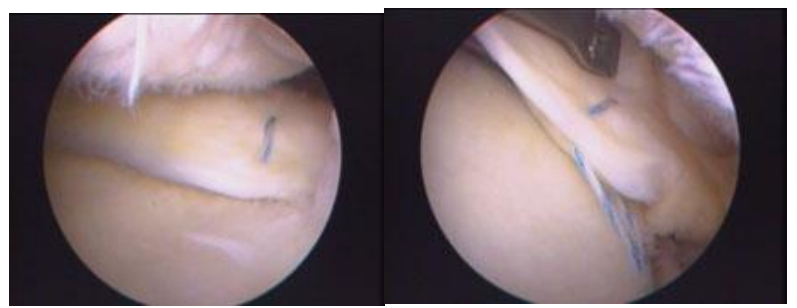

Fig. (15): Arthroscopic photography from antrolateral portal showing another 2-0 suture material was loaded, passed half centimeter medial to the tension suture

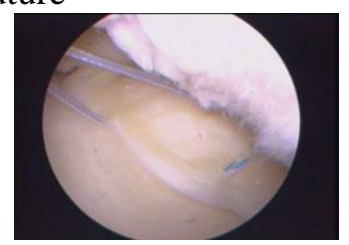

Fig. (16): Arthroscopic photography from antrolateral portal showing no 2 fibrewire was shuttelled into the meniscus

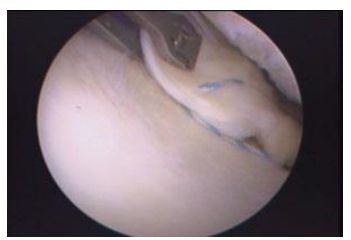

Fig. (17): Arthroscopic photography from antrolateral portal showing another no 2-0 was passed in the peripheral zone of the meniscus in line with the second fibre wire

A ring grasper was used to retrive the two inferior limbs of both the fibrewire and no. 2-0 suture material. Then, the fibre wire is shuttelled into the meniscus using the no. 2-0 suture to form a reduction suture.

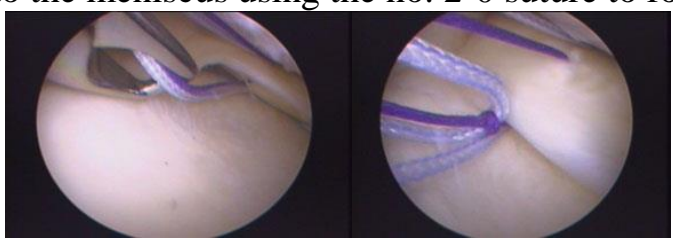

Fig. (18): Arthroscopic photography from antrolateral portal showing a ring grasper was used to retrive the two inferior limbs of both the fibre wire.

By pulling the ends of the suture material under adequate tension, the posterior root of the medial meniscus could be reduced and stabilized with adequate tension.

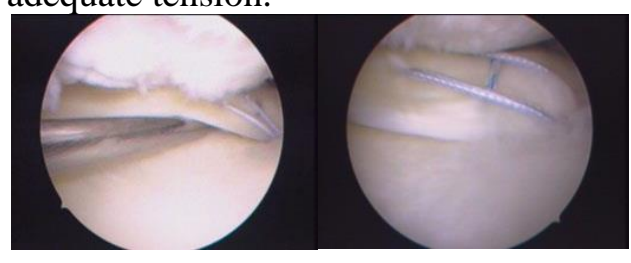


Fig. (19): Arthroscopic photography from antrolateral portal showing pulling the ends of the suture material under adequate tension.

With adequate reduction and tension, the fibre wire suture material were post-tied to and fixed with titanum endobutton on the lateral cortex of the tibia.

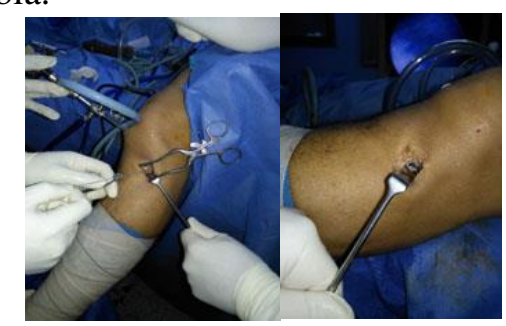

Fig. (20): Intraoperative photography showing the fibre wire suture material were post-tied to and fixed with titanum endobutton on the lateral cortex of the tibia with adequate reduction and tension.

Post_operative x ray aneteroposterior and lateral view showing endbutton just distal to jerdys tubercle.

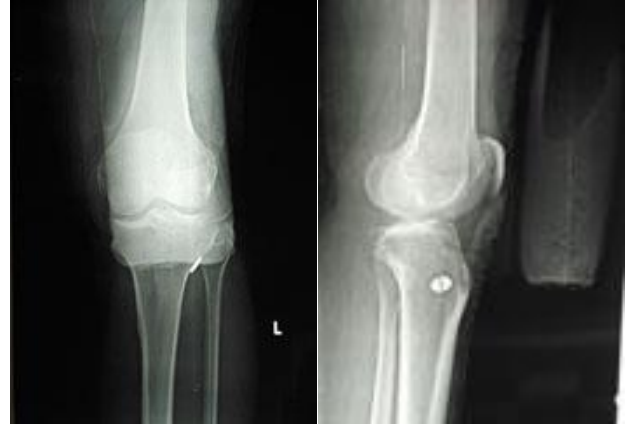

Fig. (21): Postoperative $X$ ray showing position of the endobutton just distal to jerdy's tubercle.

\section{Closure}

Subdermal skin was closed with $2-0$ or $3-0$ absorbable suture. Skin was closed with a 2-0 suture in a running or interrupted fashion, taking care to evert the skin edges. A compression dressing was applied.

\section{Postoperative care:}

The patient remained in the hospital overnight, and prophylactic parenteral antibiotics were administered for the first 24 hours postoperatively.

Postoperatively, patients visited the Outpatient Clinic one week postoperative for dressing, two weeks postoperative for stitches removal, 6 weeks postoperative for follow up of range of motion then monthly visit until 6 months postoperative. Follow up MRI was done at 6 months postoperative. Patients were non-weight bearing on crutches for 6 weeks. Patients were advised to perform quadriceps muscle exercise, as well as straight-leg raise exercises several times daily. Patients were allowed active motion of up to $90^{\circ}$ after the first 4 weeks. Then, patients were allowed to gradually increased flexion degrees up to $120^{\circ}$ after 6 weeks. Full flexion and squatting were allowed 6 months after the surgery. Patients returned to exercise after 6 months postoperative.

\section{RESULT}

Clinical outcome measures significantly improved after surgery, preoperatively according to lysholm score, 13 patients $(65 \%)$ had poor score and 7 patients $(35 \%)$ had fair score. Postoperatively, 3 patients $(15 \%)$ had poor score, 4 patients $(20 \%)$ had fair score, 6 patients (30\%) had good score and 7 patients $(35 \%)$ had excellent score (table 1). The mean IKDC score for meniscal repair increased from $60.62 \pm 5.9$ to $81.79 \pm 3.06(\mathrm{P}<0.001)$, and the mean IKDC score for partial meniscectomy increased from $52.85 \pm 6.53$ to $73.55 \pm 6.81(\mathrm{P}<0.001)$. Postoperative MRI showed complete healing in 12 patients $(60.0 \%)$ and failure in 8 patients (40.0\%) as shown in table (2). 
Table (1): Preoperative and postoperative Lysholm score

\begin{tabular}{|l|l|c|c|c|c|}
\hline \multicolumn{2}{|c|}{} & \multicolumn{2}{c|}{ Repair } & \multicolumn{2}{c|}{ Partial } \\
\cline { 3 - 6 } & Count & Percent & Count & Percent \\
\hline \multirow{4}{*}{ Preoperative } & Poor & 6 & $60 \%$ & 7 & $70 \%$ \\
\cline { 2 - 6 } & Fair & 4 & $40 \%$ & 3 & $30 \%$ \\
\cline { 2 - 6 } & Good & 0 & $0 \%$ & 0 & $0 \%$ \\
\cline { 2 - 6 } & Excellent & 0 & $0 \%$ & 0 & $0 \%$ \\
\hline \multirow{5}{*}{ Postoperative } & Poor & 0 & $0 \%$ & 3 & $30 \%$ \\
\cline { 2 - 6 } & Fair & 0 & $0 \%$ & 4 & $40 \%$ \\
\cline { 2 - 6 } & Good & 5 & $50 \%$ & 1 & $10 \%$ \\
\cline { 2 - 6 } & Excellent & 5 & $50 \%$ & 2 & $20 \%$ \\
\hline
\end{tabular}

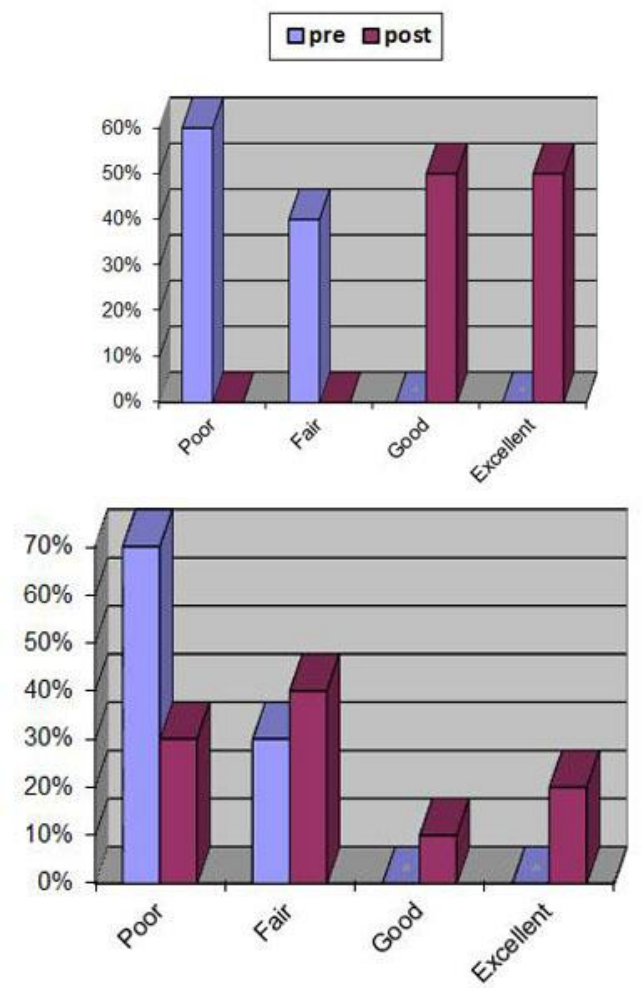

Fig. (22): Preoperative and postoperative lysholm score.

\section{IKDC:}

IKDC improved by mean $81.89 \pm 8.48$.

Table (2): Preoperative and postoperative IKDC

\begin{tabular}{|l|l|c|c|c|c|c|c|}
\hline \multicolumn{2}{|c|}{} & Mean & $\begin{array}{c}\text { Standard } \\
\text { Deviation }\end{array}$ & Median & Minimum & Maximum & $\begin{array}{c}\text { P } \\
\text { value }\end{array}$ \\
\hline \multirow{2}{*}{ Repair } & $\begin{array}{l}\text { Preoperative } \\
\text { IKDC SCORE }\end{array}$ & 60.62 & 5.9 & 59.7 & 52.8 & 68.9 & \multirow{2}{<}{} \\
\cline { 2 - 8 } & $\begin{array}{l}\text { Postoperative } \\
\text { IKDC SCORE }\end{array}$ & 81.79 & 3.06 & 84.45 & 80.4 & 89.6 & 0.001 \\
\hline \multirow{2}{*}{$\begin{array}{l}\text { Partial } \\
\text { Meniscectomy }\end{array}$} & $\begin{array}{l}\text { Preoperative } \\
\text { IKDC SCORE }\end{array}$ & 52.85 & 6.53 & 54 & 43.6 & 59.8 & \\
\cline { 1 - 7 } & $\begin{array}{l}\text { Postoperative } \\
\text { IKDC SCORE }\end{array}$ & 73.55 & 6.81 & 74.1 & 63.2 & 81.6 & \\
\hline
\end{tabular}



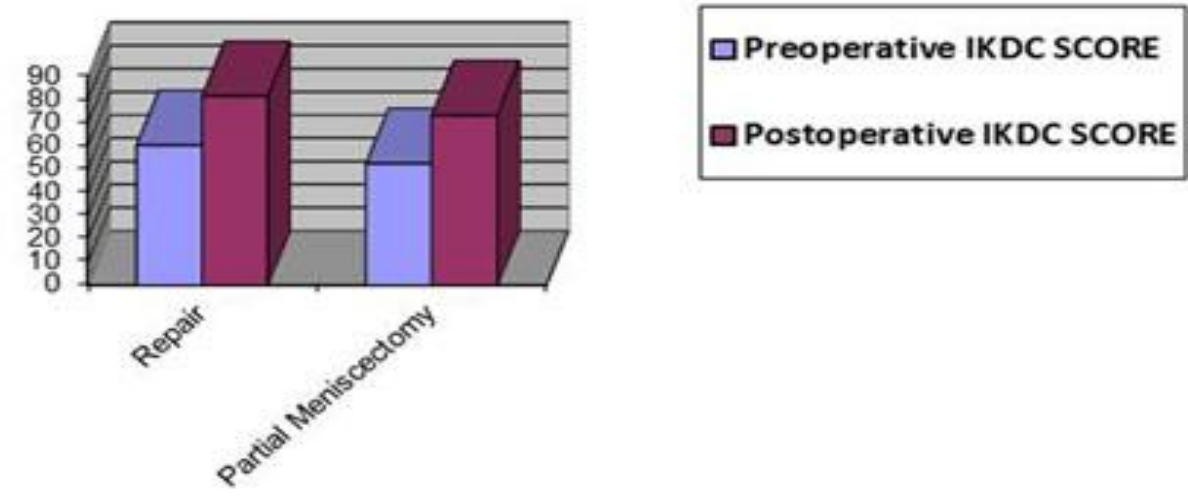

Fig. (23): Preoperative and postoperative IKDC.

\section{DISCUSSION}

Treatment of root tears has been focused on arthroscopic partial meniscectomy or repair since conservative treatment fails to restore native anatomy, which may induce arthritic changes over time (11). However, certain patients could be treated properly by conservative treatment ${ }^{(12)}$ and the clinical outcomes of conservative treatment have been reported to be competent with early diagnosis and proper treatment protocols ${ }^{(13)}$. If conservative treatment of root tears failed, patients might miss proper chance for meniscus repair. Therefore, the patient's pain might increase and arthritic change might be aggravated as time goes by ${ }^{(14)}$.

Repair restores peak contact pressure to normal ${ }^{(15)}$.

Symptomatic relief but progression of a radiographic grade of osteoarthritis after partial meniscectomy of MRT have been reported (16). Therefore, increasing attention is being paid to MRT repair and as a result, various techniques have been developed ${ }^{(17)}$. Recently, clinical studies on arthroscopic pullout repair of the MRT have reported favorable clinical and radiographic results ${ }^{(18)}$. In particular, the chondroprotective effect of repair appears to be encouraging. Kim et al $_{\mathbf{}^{(\mathbf{1 9 )}}}$ reported that MRT repair provided significantly better clinical and radiological results than partial meniscectomy and that medial meniscus extrusion decreased after repair, as determined by magnetic resonance imaging (MRI). However, it is difficult to obtain definitive information on clinical and MRI results after MRT repair because of the few studies conducted on the subject. Furthermore, the prognostic factors of repair have not been determined. Accordingly, we undertook to document the clinical and MRI results of arthroscopic pullout MRT repair and to identify the factors associated with poor prognosis.
Ten patients underwent repair with pullout suture, 10 patients were underwire partial meniscectomy due to degenerated meniscus, 20

patients included in this study were followed up to 6 months.

All clinical outcome measures significantly improved after surgery according to lysholm score where 13 patients $(65 \%)$ had poor score preoperatively and 7 patients (35\%) had fair score. Postoperatively, 3 patients (15\%) had poor score, 4 patients $(20 \%)$ had fair score, 6 patients $(30 \%)$ had good score and 7 patients (35\%) had excellent score. The mean IKDC score for meniscal repair increased from $60.62 \pm 5.9$ to $81.79 \pm$ $3.06(\mathrm{P}<0.001)$, and the mean IKDC score for partial meniscectomy increased from $52.85 \pm 6.53$ to $73.55 \pm$ $6.81(\mathrm{P}<0.001)$. Postoperative MRI, showed complete healing in 12 patients $(60.0 \%)$ and failure in 8 patients (40.0\%). Preoperatively, ME was $>3 \mathrm{~mm}$ in 17 patients $(85.0 \%)$ and $\leq 3 \mathrm{~mm}$ in 3 patients $(15.0 \%)$ that improved postoperatively to 11 patients $(55.0 \%) \leq 3 \mathrm{~mm}$ and 9 patients $(45.0 \%)>3 \mathrm{~mm}$.

Kim et al. ${ }^{(19)}$ reported that the Lysholm score improved significantly from 56.8 to 85.1 . Lee $\boldsymbol{e t}$ al. ${ }^{(18)}$ reported that the mean Lysholm knee score improved from 57.0 to 93.1 . Hong-Kyo et al. ${ }^{(20)}$ reported that the mean Lysholm score increased from 48.3 preoperatively to 83.2 postoperatively.

Chung et al. (21) compared clinical and radiological outcomes between a medial meniscus root repair cohort and a partial meniscectomy cohort at a follow-up of at least 5 years, reporting significantly better Lysholm and International Knee Documentation Committee (IKDC) scores in the repair group than in the partial meniscectomy group. Chung et al. ${ }^{(22)}$ reported that meniscal root tear repair, significantly improved in postoperative clinical subjective scores compared to preoperative status but the progression of arthrosis was not prevented and meniscal extrusion was not reduced. 


\section{CONCLUSION}

Paramount treatment for meniscal root tears historically was focused on partial menisectomy, which has recently been found to induce an unacceptable high rate of arthritic progression postoperatively. Therefore, repair of meniscus root attachments has increased in popularity and when done properly, in selected patients, demonstrate a high rate of healing as well as biomechanical and clinical efficacy in restoring the innate ability of the meniscus to dissipate axial tibiofemoral loads, thereby slowing or halting arthritic progression.

\section{REFERENCES}

1. Lee SY, Jee WH, Kim JM (2008): Radial tear of the medial meniscal root: reliability and accuracy of MRI for diagnosis. AJR Am J Roentgenol., 191: 81-5.

2. Johannsen AM, Civitarese DM, Padalecki JR et al. (2012): Qualitative and quantitative anatomic analysis of the posterior root attachments of the medial and lateral menisci. Am J Sport Med., 40: 2342-7.

3. Feucht MJ, Minzlaff P, Saier T et al. (2015): Avulsion of the anterior medial meniscus root: case report and surgical technique. Knee Surgery, Sports Traumatology, Arthroscopy, 23 (1): 146-51.

4. Sta "rke C, Kopf S, Gro"bel KH et al. (2010): The effect of a nonanatomic repair of the meniscal horn attachment on meniscal tension: a biomechanical study. Arthroscopy, 26: 358-365.

5. Choi SH, Bae S, Ji SK et al. (2012): The MRI findings of meniscal root tear of the medial meniscus: emphasis on coronal, sagittal and axial images. Knee Surg Sports Traumatol Arthrosc., 20: 2098-103.

6. Han SB, Shetty GM, Lee DH et al. (2010): Unfavorable results of partial meniscectomy for complete posterior medial meniscus root tear with early osteoarthritis: a 5to 8-year follow-up study. Arthroscopy, 26: 1326-32.

7. Kim YM, Rhee KJ, Lee JK et al. (2006): Arthroscopic pullout repair of a complete radial tear of the tibial attachment site of the medial meniscus posterior horn. Arthroscopy, 22 (7): 795.e1-795.e4.

8. Jung YH, Choi NH, Oh JS et al. (2012): All-inside repair for a root tear of the medial meniscus using a suture anchor. Am. J Sports Med., 40: 1406-1411.

9. Aagaard H, Verdonk R (1999): Function of the normal meniscus and consequences of meniscal resection. Scand-J-Med-Sci- Sports, 9 (3): 134-140.

10. Wayne H, Steven GW, Duncan R (2009): Validity of the mcmurrays test and modified versions of the test:A
Systematic Literature Review. The Journal of Manual and Manipulative Therapy, 17 (1): 22-35.

11.Hein CN, Deperio JG, Ehrensberger MT et al. (2011): Effects of medial meniscal posterior horn avulsion and repair on meniscal displacement. Knee, 18: 189-192.

12. Breitenseher MJ, Trattnig S, Dobrocky I et al. (1997): MR imaging of meniscal subluxation in the knee. Acta Radiol., 38: 876- 879.

13. Shelbourne KD, Roberson TA, Gray T (2011): Longterm evaluation of posterior lateral meniscus root tears left in situ at the time of anterior cruciate ligament reconstruction. Am J Sports Med., 39 (7): 1439-1443.

14. Krych AJ, Reardon PJ, Johnson NR et al. (2016): Non-operative management of medial meniscusu posterior horn root tears is associated with worsening arthritis and poor clinical outcome at 5-year follow-up. Knee Surg Sports TraumatolArthrosc., doi:10.1007/s00167-016-4359-8.

15.Seo JH, Li G, Shetty GM et al. (2009): Effect of repair of radial tears at the root of the posterior horn of the medial meniscus with the pullout suture technique: a biomechanical study using porcine knees. Arthroscopy, 25: $1281-1287$

16. Ozkoc G, Circi E, Gonc U et al. (2008): Radial tears in the root of the posterior horn of the medial meniscus. Knee Surg Sports TraumatolArthrosc., 16 (9): 849-854.

17.Ahn JH, Wang JH, Yoo JC et al. (2007): A pull out suture for transection of the posterior horn of the medial meniscus: using a posterior trans-septal portal. Knee Surg Sports TraumatolArthrosc., 15 (12): 1510-1513.

18. Lee JH, Lim YJ, Kim KB et al. (2009): Arthroscopic pull out suture repair of posterior root tear of the medial meniscus: radiographic and clinical results with a 2-year follow-up. Arthroscopy, 25 (9): 951-958.

19. Kim SB, Ha JK, Lee SW et al. (2011): Medial meniscus root tear refixation: comparison of clinical, radiologic, and arthroscopic findings with medial meniscectomy. Arthroscopy, 27: 346-354.

20.Hong-Kyo M, Yong-Gon $\mathrm{K}$, Yong-Chan $\mathrm{K}$ et al. (2012):Prognostic Factors of Arthroscopic Pull-out Repair for a Posterior Root Tear of the Medial Meniscus.DOI: 10.1177/0363546511435622.

21. Chung KS, Ha JK, Yeom CH et al. (2015): Comparison of clinical and radiologic results between partial meniscectomy and refixation of medial meniscus posterior root tears: a minimum 5-year follow-up. Arthroscopy, 31 (10): 1941-50.

22. Chung KS, Ha JK, Ra HJ et al. (2016): A meta-analysis of clinical and radiographic outcomes of posterior horn medial meniscus root repairs. Knee Surg Sports Traumatol Arthrosc., 24 (5): 1455-68. 